\title{
STOCHASTIC INTEGRAL REPRESENTATION OF MULTIPLICATIVE OPERATOR FUNCTIONALS OF A WIENER PROCESS
}

\author{
BY \\ MARK A. PINSKY $\left(^{1}\right)$
}

\begin{abstract}
Let $M$ be a multiplicative operator functional of $(X, L)$ where $X$ is a $d$-dimensional Wiener process and $L$ is a separable Hilbert space. Sufficient conditions are given in order that $M$ be equivalent to a solution of the linear Itô equation

$$
M(t)=I+\sum_{j=1}^{d} \int_{0}^{t} M(s) B,(x(s)) d x_{j}(s)+\int_{0}^{t} M(s) B_{0}(x(s)) d s,
$$

where $B_{0}, \ldots, B_{d}$ are bounded operator functions on $R^{d}$. The conditions require that the equation $T(t) f=E[M(t) f(x(t))]$ define a semigroup on $L^{2}\left(R^{d}\right)$ whose infinitesimal generator has a domain which contains all linear functions of the coordinates $\left(x_{1}, \ldots, x_{d}\right)$. The proof of this result depends on an a priori representation of the semigroup $T(t)$ in terms of the Wiener semigroup and a first order matrix operator. A second result characterizes solutions of the above Itô equation with $B_{0}=0$. A sufficient condition that $M$ belong to this class is that $E[M(t)]$ be the identity operator on $L$ and that $M(t)$ be invertible for each $t>0$. The proof of this result uses the martingale stochastic integral of $\mathrm{H}$. Kunita and $\mathrm{S}$. Watanabe.
\end{abstract}

1. Introduction. Let $\left\{B_{j}(x), 0 \leqq j \leqq d\right\}$ be bounded $N \times N$ matrix-valued functions on Euclidean $R^{d}$. The solution of the Cauchy problem

$$
\begin{aligned}
\frac{\partial u}{\partial t} & =\frac{1}{2} \Delta u+\sum_{j=1}^{d} B_{j}(x) \frac{\partial u}{\partial x_{j}}+B_{0}(x) u, \quad t>0, \quad x \in R^{d}, \\
u(0, x) & =f(x),
\end{aligned}
$$

can be represented as an integral over the paths of the Wiener process by the formula

$$
u(t, x)=E_{x}[M(t) f(x(t))]
$$

where $f=\left(f_{1}(x), \ldots, f_{N}(x)\right), u=\left(u_{1}(t, x), \ldots, u_{N}(t, x)\right) ; M(t)$ is the solution of the Itô stochastic integral equation ( $I=$ identity matrix)

$$
M(t)=I+\sum_{j=1}^{d} \int_{0}^{t} M(u) B_{j}(x(u)) d x_{j}(u)+\int_{0}^{t} M(u) B_{0}(x(u)) d u .
$$

These results were arrived at independently by D. G. Babbitt [1] and D. W. Stroock [9]. Stroock observed, in addition, that $M$ satisfies the multiplicative law

$$
M(t+s, \omega)=M(t, \omega) M\left(s, \theta_{t} \omega\right)
$$

Presented to the Society, June 14, 1971; received by the editors June 17, 1971.

AMS 1970 subject classifications. Primary 60H20, 60J65; Secondary 60J35, 60J55, 35K45.

Key words and phrases. Multiplicative operator functional, martingale stochastic integral, Hilbert-Schmidt operators, parabolic systems of equations.

(1) Research supported by the National Science Foundation Grant GP-9437.

Copyright (C) 1972, American Mathematical Society 
where $\theta_{t}$ denotes the shifted path. We take (1.4) as our point of departure and ask the question: Does (1.4) imply (1.3)? This is the subject of the present paper.

If $N=1$, the formula (1.2) includes the classical Feynman-Kac formula $\left(B_{j}=0\right.$ for $j \neq 0)$ and the Cameron-Martin formula $\left(B_{0}=0\right)$. In the general scalar case $(N=1)$, representation theorems for multiplicative functionals can be inferred from the work of H. Tanaka [10] and A. D. Wentsell [11]; any continuous multiplicative functional of the one-dimensional Wiener process admits the representation

$$
m(t)=\frac{f\left(x_{t}\right)}{f\left(x_{0}\right)} \exp \int_{0}^{t} g\left(x_{s}\right) d x(s)
$$

where $f$ is continuous and $g$ is locally square integrable. The form (1.5) is more general than solutions of (1.3); they coincide if $f$ has two continuous derivatives. In another direction K. Itô and S. Watanabe [3] obtained a multiplicative decomposition for multiplicative functionals of a Markov process. Their representation does not involve stochastic integrals, however.

The main problem in the case $N>1$ is lack of commutativity; since an explicit representation such as (1.5) is apparently impossible, we settle for a local characterization in terms of stochastic integrals. The first problem is to characterize "smoothness" of $t \rightarrow M(t)$. Using semigroup methods we can prove (Theorem 4.2) that a continuous solution of (1.4) comes from a solution of (1.3) whenever the formula (1.2) defines a semigroup of operators on $L^{2}\left(R^{d}\right)$ whose infinitesimal generator has a domain which contains all linear functions of the coordinates, in a suitable sense.

To obtain representation theorems in nonsmooth cases, we impose the further condition

$$
E_{x}[M(t)]=I
$$

which ensures that $t \rightarrow M(t)$ is a vector-valued martingale. Using the martingale stochastic integral of $\mathrm{H}$. Kunita and S. Watanabe [4] we prove (Theorem 5.2) that if $M(t)$ is invertible and satisfies a mild second moment condition, then it is given by a solution of (1.3) where, of course, $B_{0}=0$. The method of proof was suggested by recent work of $C$. Doléans-Dade [2].

As a by-product of the methods used to solve (1.3) it follows that all of the results stated above remain true if, instead of $N \times N$ matrices, we work with Hilbert-Schmidt operators on a Hilbert space. Using the representation (2.3) of the operator norm in terms of Hilbert-Schmidt norms, we can solve (1.3) whenever the coefficient matrices are replaced by bounded operators. In particular, the representation formula (1.2) applies to solve certain partial differential equations with operator coefficients.

Finally, we mention that the results of this paper constitute one aspect of the general problem of representation of multiplicative operator functionals of a Markov process. These were introduced in [8], where representation theorems were 
given in the case the underlying Markov process is a chain with a finite number of states. From the point of view of random evolutions, the novelty of the problems under consideration here is the appearance of the operators $B_{1}(x), \ldots, B_{d}(x)$; their effect is to intertwine the Markov process in a nontrivial way with the multiplicative functional.

$\$ 2$ contains notational preliminaries on stochastic integrals and Hilbert-Schmidt operators. $\S 3$ contains an extension of the Babbitt-Stroock solution of the stochastic integral equation (1.3) in the case $\left\|B_{j}\right\|$ is bounded by a constant independent of $x$, $0 \leqq j \leqq d$. Their construction is further extended to the more natural class for which $B_{0}(x)$ is locally integrable and $B_{j}(x)$ is locally square integrable, $1 \leqq j \leqq d$. In $\S 4$, we give the representation theorem in the case $\left\|B_{j}\right\|$ bounded, $t \rightarrow M(t)$ smooth. The main tool used in the proof is the a priori derivation of the integral equation (4.5); this equation, in turn, uniquely determines the semigroup (1.2). $\$ 5$ contains the results on martingale multiplicative functionals.

2. Notation. $R^{d}$ will denote the real $d$-dimensional Euclidean space of vectors $x=\left(x_{1}, \ldots, x_{d}\right) . X=\left(x(t), \mathscr{F}_{t}, P_{x}\right)$ is a Wiener process on $R^{d}$ with transition semigroup

$$
T^{0}(t) f(x)=\int_{R_{d}} \frac{\exp \left(-(x-y)^{2} / 2 t\right)}{(2 \pi t)^{d / 2}} f(y) d y .
$$

This semigroup is a contraction on $L^{2}\left(R^{d}\right)$. $\mathscr{F}_{t}=\sigma(x(s) ; s \leqq t)$.

$L$ will denote a separable Hilbert space with inner product $\langle\rangle,,|x|=\langle x, x\rangle^{1 / 2}$. (Although we treat only real scalars, the complex case presents no difficulties.) $\tilde{L}=L^{2}\left(R^{d} ; L\right)$ is the Hilbert space of all measurable, $L$-valued functions $x \rightarrow \tilde{f}(x)$, equipped with the norm $\|\tilde{f}\|=\left\{\int_{R_{d}}|\tilde{f}(x)|^{2} d x\right\}^{1 / 2} . \mathscr{M}$ is the collection of all linear operators $A$ on $L$ with finite Hilbert-Schmidt norm:

$$
\|A\|_{2}^{2}=\sum_{k \geqq 1}\left|A \varphi_{k}\right|^{2}<\infty,
$$

where $\left\{\varphi_{k}\right\}_{k \geq 1}$ is an orthonormal basis for $L$. It is easily checked that this norm is well defined, independent of the basis. We have the inequalities

$$
\|A+B\|_{2} \leqq\|A\|_{2}+\|B\|_{2}
$$

and

$$
\|A B\|_{2} \leqq\|A\|_{2}\|B\|_{2} .
$$

These show, in particular, that $\mathscr{M}$ is an algebra; $\mathscr{M}$ is also a Hilbert space.

The standard operator norm is defined by $\|A\|_{\text {op }}=\sup _{|x|=1}|A x|$. Let $\mathscr{L}$ be the algebra of all bounded operators. It is easily checked that the two norms are related by the estimate

$$
\|A B\|_{2} \leqq\|A\|_{\text {op }}\|B\|_{2}, \quad B \in \mathscr{M}, \quad A \in \mathscr{L} .
$$

The following makes this precise:

(2.3) Proposition. $\|A\|_{\text {op }}=\sup _{0 \neq B \in \mathscr{M}}\|A B\|_{2} /\|B\|_{2}$. 
Proof. Let the right-hand side be denoted by $a$. Let $\left\{x_{n}\right\}_{n \geqq 1}$ be a sequence of vectors with $\left|x_{n}\right|=1,\left|A x_{n}\right| \rightarrow\|A\|_{\text {op }}$. Let $B=B_{n}$ be the orthogonal projection on $x_{n}: B x=\left\langle x, x_{n}\right\rangle x_{n}$. Thus $\|A B\|_{2}^{2}=\sum_{k}\left|A B \varphi_{k}\right|^{2}=\sum_{k}\left\langle\varphi_{k}, x_{n}\right\rangle^{2}\left|A x_{n}\right|^{2}=\left|A x_{n}\right|^{2}$; but $\|B\|_{2}^{2}=1$; thus $\|A B\|_{2} /\|B\|_{2}=\left|A x_{n}\right| \rightarrow\|A\|_{\text {op }}$ and hence $a \geqq\|A\|_{\text {op. }}$. But $a \leqq\|A\|_{\text {op }}$ by $\left(2.2^{\prime}\right)$. Thus the conclusion follows.

If $s \rightarrow A(s)$ is a nonanticipating $\left({ }^{2}\right)$ functional of $X$ with values in $\mathscr{M}$, with $P_{x}\left[\int_{0}^{t}\|A(s)\|_{2}^{2} d s<\infty\right]=1$, we can define the Itô stochastic integral $\int_{0}^{t} A(s) d x_{j}(s)$ as that element $B$ of $\mathscr{M}$ such that $\left\langle B \varphi_{k}, \varphi_{l}\right\rangle=\int_{0}^{t}\left\langle A(s) \varphi_{k}, \varphi_{l}\right\rangle d x_{j}(s)(k, l \geqq 1)$, relative to a basis $\left\{\varphi_{k}\right\}$. It is easily checked that $B$ is thus well defined; $x_{j}(t)$ is the $j$ th component of $x(t)$.

The following formula gives a convenient bound on the map $A \rightarrow B$,

$$
E_{x}\left[\left\|\int_{0}^{t} A(s) d x_{j}(s)\right\|_{2}^{2}\right]=\int_{0}^{t} E_{x}\|A(s)\|_{2}^{2} d s
$$

whenever the right-hand side is finite. To prove this, simply compute the HilbertSchmidt norm indicated on the left-hand side in terms of an orthonormal basis and use the classical relation

$$
E_{x}\left[\left(\int_{0}^{t} a(s) d x(s)\right)^{2}\right]=E_{x}\left[\int_{0}^{t} a(s)^{2} d s\right] .
$$

Equation (2.4) is the central reason for use of the Hilbert-Schmidt norm (2.0). Presumably the ordinary operator norm $\|A\|_{\text {op }}=\sup _{|x|=1}\|A x\|$ does not satisfy (2.4), even if we replace $=$ by $\leqq$.

A multiplicative operator functional (MOF) of $(X, L)$ is a mapping $(t, \omega)$ $\rightarrow M(t, \omega)$ from $[0, \infty) \times \Omega$ to bounded operators on $L$ which possess the following properties:

(i) $\omega \rightarrow M(t, \omega) f$ is $\mathscr{F}_{t}$ measurable, $t>0, f \in L$.

(ii) $t \rightarrow M(t, \omega) f$ is right continuous a.s., $f \in L$.

(iii) $M(t+s, \omega)=M(t, \omega) M\left(s, \theta_{t} \omega\right)$ a.s. for each $s, t \geqq 0$.

(iv) $M(0, \omega)=I$, the identity operator on $L$.

If for each pair $(t, \omega), M(t, \omega)$ is a Hilbert-Schmidt operator on $L$, we will speak of an $\mathscr{M}$-valued MOF of $(X, L)$. Otherwise $M$ will be an $\mathscr{L}$-valued MOF of $(X, L)$. The expectation semigroup is the family of operators $\{T(t), t \geqq 0\}$ where $T(t) f(x)$ $=E_{x}[M(t) f(x(t))]$.

3. Solution of a stochastic integral equation. The following theorem gives the natural extension of the construction of Babbitt and Stroock.

(3.0) Theorem. Let $X$ be a Wiener process on $R^{d}$ and let $B_{0}(s), \ldots, B_{d}(s)$ be nonanticipating, $\mathscr{L}$-valued functionals with

$$
\sup _{s \geqq 0: \omega \in \Omega ; 0 \leqq j \leqq d}\left\|B_{j}(s, \omega)\right\|_{\mathrm{op}} \leqq B<\infty
$$

$\left.{ }^{2}\right) \forall t,\{\omega: A(t, \omega) \in U\} \in \mathscr{F}_{t}$ if $U$ is a Borel set in $\mathscr{M}$. 
Let $A \in \mathscr{M}, A \in \mathscr{F}_{\text {s. The equation }}$

$$
M(t)=A+\sum_{j=1}^{d} \int_{s}^{t} M(u) B_{j}(u) d x_{j}(u)+\int_{s}^{t} M(u) B_{0}(u) d u \quad(t \geqq s)
$$

has a unique, continuous, $\mathscr{M}$-valued nonanticipating solution $t \rightarrow M_{A}(t)$. This solution satisfies

$$
E_{x}\left\|M_{A}(t)\right\|_{2}^{2} \leqq 2 \exp \left\{2 B^{2}(d+1)(t-s)\right\} E_{x}\|A\|_{2}^{2}
$$

Proof. Let

$$
\begin{aligned}
R_{0}(t) & =A \\
R_{n+1}(t) & =\sum_{j=1}^{d} \int_{s}^{t} R_{n}(u) B_{j}(u) d x_{j}(u)+\int_{s}^{t} R_{n}(u) B_{0}(u) d u .
\end{aligned}
$$

By the triangle inequality, we have

$$
\begin{aligned}
\left\{E_{x}\left\|R_{n+1}(t)\right\|_{2}^{2}\right\}^{1 / 2} \leqq & \sum_{j=1}^{d}\left\{E_{x}\left\|\int_{s}^{t} R_{n}(u) B_{j}(u) d x_{j}(u)\right\|_{2}^{2}\right\}^{1 / 2} \\
& +\left\{E_{x}\left\|\int_{s}^{t} R_{n}(u) B_{0}(u) d u\right\|_{2}^{2}\right\}^{1 / 2}
\end{aligned}
$$

Thus by (2.4)

$$
\begin{aligned}
\left\{E_{x}\left\|R_{n+1}(t)\right\|_{2}^{2}\right\}^{1 / 2} & \leqq B \sum_{j=1}^{d}\left\{\int_{s}^{t} E_{x}\left\|R_{n}(u)\right\|_{2}^{2} d u\right\}^{1 / 2}+B\left\{\int_{s}^{t} E_{x}\left\|R_{n}(u)\right\|_{2}^{2} d u\right\}^{1 / 2} \\
& =B(d+1)\left\{\int_{s}^{t} E_{x}\left\|R_{n}(u)\right\|_{2}^{2} d u\right\}^{1 / 2} .
\end{aligned}
$$

Upon iteration this yields

$$
E_{x}\left[\left\|R_{n}(t)\right\|_{2}^{2}\right] \leqq\left(\left[B^{2}(d+1)^{2}(t-s)\right]^{n} / n !\right) E_{x}\|A\|_{2}^{2} .
$$

Hence by the martingale inequality,

$$
\begin{aligned}
P_{x}\left\{\max _{s \leqq t \leqq T}\left\|R_{n+1}(t)-\int_{s}^{t} R_{n}(u) B_{0}(u) d u\right\|_{2} \geqq \lambda\right\} \\
\leqq \frac{1}{\lambda^{2}} E_{x}\left\{\left\|\sum_{j=1}^{d} \int_{s}^{T} R_{n}(u) B_{j}(u) d x_{j}(u)\right\|_{2}^{2}\right\} \\
\leqq\left(1 / \lambda^{2}\right) B^{2}(d+1)^{2} E_{x}\left[\left\|R_{n}(T)\right\|_{2}^{2}\right] \\
\leqq\left(B^{2}(d+1)^{2} / \lambda^{2}\right)\left(\left[B^{2}(d+1)^{2}(T-s)\right]^{n} / n !\right) E_{x}\|A\|_{2}^{2} .
\end{aligned}
$$

The choice $\lambda=2^{-n}$ gives a convergent series and hence, by the Borel-Cantelli lemma, the series $\sum_{n=0}^{\infty}\left\{R_{n+1}(t)-\int_{s}^{t} R_{n}(u) B_{0}(u) d u\right\}$ converges uniformly for $s \leqq t \leqq T$. On the other hand,

$$
\begin{aligned}
P_{x}\left\{\max _{s \leqq t \leqq T}\left\|\int_{s}^{t} R_{n}(u) B_{0}(u)\right\|_{2} \geqq \lambda\right\} & \leqq P_{x}\left\{\int_{s}^{T}\left\|R_{n}(u) B_{0}(u)\right\|_{2} d u \geqq \lambda\right\} \\
& \leqq \frac{1}{\lambda} E_{x}\left[\int_{s}^{T}\left\|R_{n}(u) B_{0}(u)\right\|_{2} d u\right] \\
& \leqq \frac{B}{\lambda} \int_{s}^{T} E_{x}\left[\left\|R_{n}(u)\right\|_{2}^{2}\right]^{1 / 2} d u \\
& \leqq(\text { const })^{n} /(n !)^{1 / 2} \quad\left(\lambda=2^{-n}\right) .
\end{aligned}
$$


Hence the series $\sum_{n=0}^{\infty} \int_{s}^{t} R_{n}(u) B_{0}(u) d u$ converges uniformly for $s \leqq t \leqq T$. Let $M_{n}(t)$ $=\sum_{k=0}^{n} R_{k}(t), M_{A}(t)=\lim _{n \uparrow \infty} M_{n}(t)$. We have

$$
M_{n+1}(t)=A+\sum_{j=1}^{d} \int_{s}^{t} M_{n}(u) B_{j}(u) d x_{j}(u)+\int_{s}^{t} M_{n}(u) B_{0}(u) d u
$$

the second integral clearly converges to $\int_{s}^{t} M(u) B_{0}(u) d u$. As for the first integral, we have

$$
\begin{aligned}
P_{x}\left\{\max _{s \leqq t \leqq T} \| \sum_{j=1}^{d} \int_{s}^{t}\left\{M(u)-M_{n}(u)\right\} B_{j}(u)\right. & \left.d x_{j}(u) \|_{2} \geqq \lambda\right\} \\
& \leqq \frac{B^{2}}{\lambda^{2}}(d) \int_{s}^{t} E_{x}\left[\left\|M(u)-M_{n}(u)\right\|_{2}^{2}\right] d u .
\end{aligned}
$$

By (3.3) the last term is the general term of a convergent series if $\lambda=2^{-n}$. Thus the stochastic integrals converge and we have solved (3.1).

To prove (3.2), apply the triangle inequality to (3.1) to yield

$$
E_{x}\left[\left\|M_{A}(t)\right\|_{2}^{2}\right] \leqq 2 E_{x}\|A\|_{2}^{2}+2 B^{2}(d+1)^{2} \int_{s}^{t} E_{x}\left\|M_{A}(u)\right\|_{2}^{2} d u .
$$

An application of Gronwall's lemma completes the proof.

To prove the uniqueness, let $M_{1}(t)$ and $M_{2}(t)$ be two solutions of (3.1). $M=M_{1}$ $-M_{2}, \tau_{n}=\inf \left\{t:\|M(t)\|_{2}=n\right\}, I(u)=I_{\left\{0, \tau_{n}\right\}}(u)$.

Then we have [7]

$$
M\left(t \wedge \tau_{n}\right)=\sum_{j=1}^{d} \int_{s}^{t} M(u) I(u) B_{j}(u) d x_{j}(u)+\int_{s}^{t} M(u) I(u) B_{0}(u) d u
$$

and hence

$$
E_{x}\left[\left\|M\left(t \wedge \tau_{n}\right)\right\|_{2}^{2}\right] \leqq(d+1)^{2} B^{2} \int_{s}^{t} E_{x}\left[\|M(u) I(u)\|_{2}^{2}\right] d u
$$

but the left-hand member is greater than $E_{x}\left[\|M(t) I(t)\|_{2}^{2}\right]$, and thus

$$
0=E_{x}\left[\|M(t) I(t)\|_{2}^{2}\right]=E_{x}\left[\|M(t)\|_{2}^{2} ;\|M(t)\|_{2}<n\right] .
$$

Letting $n \rightarrow \infty$ gives the result. Hence the theorem is proved.

In the next step we specialize to the case $B(u)=B(x(u))$ while at the same time replacing the initial operator by the identity (whose Hilbert-Schmidt norm is infinite!). Of key importance is the evaluation (2.3).

(3.4) Corollary. Let $B_{0}(x), \ldots, B_{d}(x)$ be bounded, $\mathscr{L}$-valued functions on $R^{d}$. The equation

$$
M(t)=I+\sum_{j=1}^{d} \int_{0}^{t} M(u) B_{j}(x(u)) d x_{j}(u)+\int_{0}^{t} M(u) B_{0}(x(u)) d u
$$

has a unique $\mathscr{L}$-valued solution $(t, \omega) \rightarrow M(t, \omega)$. It satisfies the multiplicative property $M(t, \omega)=M(s, \omega) M\left(t-s, \theta_{s}, \omega\right)$, and the bound

$$
E_{x}\|M(t)\|_{\text {op }}^{2} \leqq 2 \exp \left\{2 B^{2}(d+1)^{2} t\right\} .
$$

Proof. By the proof of Theorem (3.0), each iterate is of the form $R_{n}(t)=A S_{n}(t)$, where $S_{n}(t)$ is a bounded operator. In view of Proposition (2.3), we must have 
$\left\|S_{n}(t)\right\|_{\text {op }}^{2}=\sup _{\|A\|_{2}=1}\left\|A S_{n}(t)\right\|_{2}^{2}$; let $A_{j}=A_{j}(\omega)$ be a sequence of elements of $\mathscr{M}$ such that $\left\|A_{j}\right\|_{2}^{2}=1$, and $\left\|A_{j} S_{n}(t)\right\|_{2}$ increases monotonically to $\left\|S_{n}(t)\right\|_{\text {op }}$. Then

$$
E_{x}\left\|S_{n}(t)\right\|_{\mathrm{op}}^{2}=\lim _{j} E_{x}\left\|A_{j} S_{n}(t)\right\|_{2}^{2}=\lim _{j} E_{x}\left\|R_{n}(t)\right\|^{2} \leqq\left[B^{2}(d+1)^{2} t\right]^{n} / n !
$$

The same argument applies to $M_{A}(t)=A M(t)$. This yields (3.6). To obtain the multiplicative property, note that if $A \in \mathscr{M}, t \rightarrow M_{A}(t, \omega)$ is a solution of (3.1) with $A$ replaced by $M_{A}(s, \omega), B_{j}(u)=B_{j}(x(u))$. But a simple calculation shows that $M_{A}(s) M\left(t-s, \theta_{s}\right)$ is a solution of the same equation; hence the two are equal and the multiplicative property follows by the identification of the factors of $A$.

The boundedness assumptions can be relaxed somewhat, as the following shows. The proof is modelled on a similar argument of H. P. McKean, Jr. [7, p. 36ff].

(3.7) Proposition. Let $B_{0}(s), \ldots, B_{d}(s)$ be $\mathscr{L}$-valued nonanticipating functionals such that

$$
\int_{0}^{t}\left\{\left\|B_{0}(s)\right\|_{\mathrm{op}}+\sum_{j=1}^{d}\left\|B_{j}(s)\right\|_{\mathrm{op}}^{2}\right\} d s<\infty
$$

for each $t>0$, with probability one. Then equation (3.1) has a unique continuous nonanticipating $\mathscr{M}$-valued solution $t \rightarrow M(t)$.

Proof. We give the proof in the case $s=0$. Let

$$
f(t)=\int_{0}^{t}\left\{\left\|B_{0}(s)\right\|_{\mathrm{op}}+\sum_{j=1}^{d}\left\|B_{j}(s)\right\|_{\mathrm{op}}^{2}\right\} d s,
$$

and let $s \rightarrow T(s)$ be its inverse; we assume without loss of generality that $f(\infty)=\infty$. $T(s)=\inf \{t: f(t)=s\}$. Let

$$
\begin{aligned}
Z_{0}(t) & =A, \\
Z_{n+1}(t) & =\sum_{j=1}^{d} \int_{0}^{t} Z_{n}(u) B_{j}(u) d x_{j}(u)+\int_{0}^{t} Z_{n}(u) B_{0}(u) d u .
\end{aligned}
$$

Then $\left({ }^{3}\right)$

$$
\left\|Z_{n+1}(T(s))\right\|_{2}^{2} \leqq(d+1) \sum_{j=1}^{d}\left\|\int_{0}^{T(s)} Z_{n}(u) B_{j}(u) d x_{j}(u)\right\|_{2}^{2}+(d+1)\left\|\int_{0}^{T(s)} Z_{n}(u) B_{0}(u) d u\right\|_{2}^{2} .
$$

Thus

$$
\begin{aligned}
E_{x}\left[\left\|Z_{n+1}(T(s))\right\|_{2}^{2}\right] \leqq & (d+1) \sum_{j=1}^{d} E\left[\int_{0}^{T(s)}\left\|Z_{n}(u) B_{j}(u)\right\|_{2}^{2} d u\right] \\
& +(d+1) E\left[\int_{0}^{T(s)}\left\|Z_{n}(u)\right\|_{2}^{2}\left\|B_{0}(u)\right\|_{2} \int_{0}^{T(s)}\left\|B_{0}(u)\right\|_{2} d u\right] \\
\leqq & (d+1) E_{x}\left[\int_{0}^{T(s)}\left\|Z_{n}(u)\right\|_{2}^{2}\left\{\sum_{j=1}^{d}\left\|B_{j}(u)\right\|_{\mathrm{op}}^{2}+s\left\|B_{0}(u)\right\|_{\mathrm{op}}\right\} d u\right] \\
\leqq & (d+1) E_{x}\left[\int_{0}^{T(s)}\left\|Z_{n}(u)\right\|_{2}^{2} d f(u)\right] \\
= & (d+1) E_{x}\left[\int_{0}^{s}\left\|Z_{n}(T(\theta))\right\|_{2}^{2} d \theta\right], \quad \theta=f(u),
\end{aligned}
$$

${ }^{3}$ ) At this point we use the polar decomposition of the operator $B_{0}$, together with the Schwarz inequality. 
where we have used the fact that $s \leqq 1$. Hence we have the estimate

$$
E_{x}\left[\left\|Z_{n}(T(s))\right\|_{2}^{2}\right] \leqq((d+1) s)^{n} / n !, \quad s \leqq 1 .
$$

On the other hand, $\sum_{j=1}^{d} \int_{0}^{T(s)} Z_{n}(u) B_{j}(u) d x_{j}(u)$ is a martingale with respect to the fields $\mathscr{F}_{T(s)}$. Thus

$$
\begin{aligned}
& P_{x}\left[\max _{s \leqq t}\left\|\sum_{j=1}^{d} \int_{0}^{T(s)} Z_{n}(u) B_{j}(u) d x_{j}(u)\right\|_{2} \geqq \lambda\right] \\
& \leqq \frac{1}{\lambda^{2}} E_{x}\left[\sum_{j=1}^{d}\left\|\int_{0}^{T(t)} Z_{n}(u) B_{j}(u) d x_{j}(u)\right\|_{2}^{2}\right] \\
& \leqq \frac{1}{\lambda^{2}} E_{x}\left[\int_{0}^{t}\left\|Z_{n}(T(u))\right\|_{2}^{2} d u\right] \leqq \frac{1}{\lambda^{2}} \frac{(d+1)^{n} t^{n+1}}{(n+1) !} .
\end{aligned}
$$

If we choose $\lambda=n t^{n+1 / 2} 2^{n / 2}[(n+1) !]^{-1 / 2}$, we get by the first Borel-Cantelli lemma that

$$
P_{x}\left[\max _{s \leqq t}\left\|\sum_{j=1}^{d} \int_{0}^{T(s)} Z_{n}(u) B_{j}(u) d x_{j}(u)\right\|_{2} \leqq \frac{n t^{(n+1) / 2} 2^{n / 2}}{[(n+1) !]^{1 / 2}}, n \uparrow \infty\right]=1 .
$$

The Lebesgue integral term is estimated by

$$
\begin{aligned}
P_{x}\left[\max _{s \leqq t}\left\|\int_{0}^{T(s)} Z_{n}(u) B_{0}(u) d u\right\|_{2} \geqq \lambda\right] \\
\quad \leqq P\left[\max _{s \leqq t} \int_{0}^{T(s)}\left\|Z_{n}(u)\right\|_{2}^{2}\left\|B_{0}(u)\right\|_{\mathrm{op}} \geqq \lambda\right] \leqq \frac{1}{\lambda^{2}} 2^{n} t^{n+1} /(n+1) !
\end{aligned}
$$

Thus with the same choice of $\lambda$, we have

$$
P_{x}\left[\max _{s \leqq t}\left\|\int_{0}^{T(s)} Z_{n}(u) B_{0}(u) d u\right\|_{2} \leqq \frac{n t^{(n+1) / 2} 2^{n / 2}}{[(n+1) !]^{1 / 2}}, n \uparrow \infty\right]=1 .
$$

Let $M(t)=\sum_{n=0}^{\infty} Z_{n}(t)$. Since $f(\infty)=\infty$, this series converges uniformly on compact $t$-sets and is a solution of the equation (3.1). Thus we have solved the equation for $t \leqq 1$. But this restriction is inessential. To solve the equation for $t \leqq T_{0}$, the estimate (3.8) becomes $\left(2 T_{0}\right)^{n} / n$ ! and the remaining analysis is above.

4. Representation of smooth multiplicative functionals. In this section we seek to characterize the operator $M(t)$ of the previous section. The method leans heavily on the a priori representation (4.5) of the expectation semigroup. The quantities of interest are

$$
B_{0}(x)=\lim _{t \downarrow 0} E_{x}\left\{\frac{M(t)-I}{t}\right\}
$$

and

$$
B_{j}(x)=\lim _{t \downarrow 0} E_{x}\left\{\frac{(M(t)-I)\left(x_{j}(t)-x_{j}(0)\right)}{t}\right\}, \quad 1 \leqq j \leqq d,
$$

where the limits are taken in the operator norm of $\mathscr{L}$. The main hypothesis of this section is 
(4.1) The limits (4.1a) and (4.1b) are uniform in $x \in R^{d}$.

(4.2) THEOREM. Let $t \rightarrow M(t)$ be an $\mathscr{L}$-valued multiplicative operator functional of $(X, L)$ such that

(a) $\sup _{t \leqq T} \sup _{x} E_{x}\|M(t)\|_{\mathrm{op}}^{2}=C(T)<\infty$

(b) $t \rightarrow M(t)$ is continuous a.s. from $[0, \infty) \rightarrow \mathscr{L}$,

(c) $\lim _{h \rightarrow 0} E_{x}\left\{\|M(t+h)-M(t)\|_{\mathrm{op}}^{2}\right\}=0$ uniformly in $x \in R^{d}$,

(d) (4.1) holds.

Then for each $x \in R^{d}, P_{x}\left[M(t)=M_{1}(t), t \geqq 0\right]=1$, where $M_{1}(t)$ is the solution of equation (1.3), constructed in the previous section.

The proof is broken into several lemmas. Let

$$
T(t) \tilde{f}=E_{x}[M(t) \tilde{f}(x(t))] ; \quad T^{0}(t) \tilde{f}=E_{x}[\tilde{f}(x(t))] .
$$

(4.3) LEMMA. $T(t)$ is a semigroup on $\tilde{L}=L^{2}\left(R^{n}, L\right)$.

\section{Proof.}

$$
\begin{aligned}
\int_{R^{d}}\left|E_{x}\{M(t) \tilde{f}(x(t))\}\right|^{2} d x & \leqq \int_{R^{d}}\left(E_{x}\|M(t)\|_{\mathrm{op}}|\tilde{f}(x(t))|\right)^{2} d x \\
& \leqq \int_{R^{d}} E_{x}\|M(t)\|_{\mathrm{op}}^{2} E_{x}|\tilde{f}(x(t))|^{2} d x \\
& \leqq C(t) \int_{R^{d}} E_{x}|\tilde{f}(x(t))|^{2} d x \\
& =C(t) \int_{R^{d}}|\tilde{f}|^{2} d x
\end{aligned}
$$

Thus $T(t)$ is a bounded operator on $\tilde{L}$.

To complete the proof, we have

$$
\begin{aligned}
T(t+s) \tilde{f}(x) & =E_{x}[M(t+s) \tilde{f}(x(t+s))]=E_{x}\left[M(t) M\left(s, \theta_{t}\right) \tilde{f}\left(x\left(s, \theta_{t}\right)\right)\right] \\
& =E_{x}\left[M(t) E_{x(t)}[M(s) \tilde{f}(x(s))]\right]=T(t)(T(s) \tilde{f})(x) .
\end{aligned}
$$

In the following lemma, recall that

$$
T^{0}(t) \tilde{f}(x)=\int_{R^{d}} \tilde{f}(x+y) \frac{\exp \left(-|y|^{2} / 2 t\right)}{(2 \pi t)^{d / 2}} d y .
$$

Let $P \tilde{f}=(B \cdot \nabla) \tilde{f}+B_{0} \tilde{f}, \tilde{u}=T^{0}(t) \tilde{f}$. Since $T^{0}$ maps $L^{2}$ functions into $C^{\infty}$ functions, the composition $P T^{0}(t)$ is well defined; further we have the following estimate, whose proof in the finite dimensional case is due to Babbitt. The proof below is entirely parallel to Babbitt's.

(4.4) Lemma. $\left\|P T^{0}(t) \tilde{f}\right\| \leqq K(t)\|\tilde{f}\|$ where $K \in L^{1}[0, T]$ for each $T>0$.

Proof. $P T^{0}(t) \tilde{f}=B_{0}(x) \tilde{u}+\sum_{i=1}^{d} B_{i}(x)\left(\partial \tilde{u} / \partial x_{i}\right)$; the first term is trivially estimated 
by $\left\|B_{0}\right\|_{\infty}\|u\|_{2}$. For the second term, we apply the convolution estimate $\|a * b\|_{2}$ $\leqq\|a\|_{1}\|b\|_{2}$ to the expression

$$
\begin{aligned}
\left\|\frac{\partial \tilde{u}}{\partial x_{i}}\right\| & =\left\|\int_{R^{d}} \frac{(x-y)_{i}}{t} \frac{\exp \left(-|x-y|^{2} / 2 t\right)}{(2 \pi t)^{d / 2}} \tilde{f}(y) d y\right\| \\
& \leqq \frac{1}{t^{1 / 2}} \int_{R^{d}} \frac{\left|x_{i}-y_{i}\right|}{t^{1 / 2}} \frac{\exp \left(-|x-y|^{2} / 2 t\right)}{(2 \pi t)^{d / 2}} d y\left(\int_{R^{d}}|\tilde{f}(y)|^{2} d y\right)^{1 / 2} \\
& =(2 / \pi t)^{1 / 2}\|\tilde{f}\| .
\end{aligned}
$$

Thus $\left\|P T^{0}(t) \tilde{f}\right\| \leqq\left(\left\|B_{0}\right\|_{\infty}+\sum_{i=1}^{d}(2 / \pi t)^{1 / 2}\left\|B_{i}\right\|_{\infty}\right)\|\tilde{f}\|$ completing the proof of the lemma.

(4.5) LemMa. $T(t) \tilde{f}-T^{0}(t) \tilde{f}=\int_{0}^{t} T(s) P T^{0}(t-s) \tilde{f} d s, \tilde{f} \in \tilde{L}$.

Proof. Since both left- and right-hand sides are bounded operators on $\tilde{L}$, it suffices to prove the equation on the dense subspace consisting of those $f$ for which $\partial^{2} f / \partial x_{i} \partial x_{j}$ is bounded and $\in \tilde{L}, 1 \leqq i \leqq j \leqq d$. We have (to within $o(1)$ )

$$
\begin{aligned}
T(t) \tilde{f}-T^{0}(t) \tilde{f} & =E_{x}[(M(t)-I) \tilde{f}(x(t))] \\
& =\sum_{k=1}^{\left[2^{n} t\right]} E_{x}\left[\left(M\left(\frac{k}{2^{n}}\right)-M\left(\frac{k-1}{2^{n}}\right)\right) \tilde{f}(x(t))\right] \\
& =\sum_{k=1}^{\left[2^{n} t\right]} E_{x}\left[M\left(\frac{k-1}{2^{n}}\right)\left(M\left(\frac{1}{2^{n}}, \theta_{(k-1) / 2^{n}}\right)-I\right) \tilde{f}\left(x\left(t-\frac{k-1}{2^{n}}\right) \theta_{(k-1) / 2^{n}}\right)\right] \\
& =\sum_{k=1}^{\left[2^{n} t\right]} E_{x}\left[M\left(\frac{k-1}{2^{n}}\right) E_{x\left((k-1) / 2^{n}\right)}\left[\left(M\left(\frac{1}{2^{n}}\right)-I\right) f\left(x\left(t-\frac{k-1}{2^{n}}\right)\right)\right]\right] \\
& =\frac{1}{2^{n}} \sum_{k=1}^{\left[2^{n} t\right]} T\left(\frac{k-1}{2^{n}}\right) P_{1 / 2^{n}} T^{0}\left(t-\frac{k}{2^{n}}\right) \tilde{f}
\end{aligned}
$$

where we have set $P_{\delta} \tilde{g}(x)=E_{x}[((M(\delta)-I) / \delta) \tilde{g}(x(\delta))]$ and used the simple Markov property twice, after an initial application of the multiplicative property of $M$. Note that for $g \in \tilde{L}$,

$$
\begin{aligned}
P_{\delta} g-P T^{0}(\delta) g= & E_{x}\left[(M(\delta)-I) / \delta-B_{0}(x)\right] g(x)+P E_{x}\{g(x)-g(x(\delta))\} \\
& +E_{x}[((M(\delta)-I) / \delta)(g(x(\delta)))-g(x)-(B \cdot \nabla) g] .
\end{aligned}
$$

We now write the final expression above as I+II + III + IV, where

$$
\begin{aligned}
\mathrm{I} & =\frac{1}{2^{n}} \sum_{k=1}^{\left[2^{n}\right]} T\left(\frac{k-1}{2^{n}}\right) P T^{0}\left(t-\frac{k-1}{2^{n}}\right) \tilde{f}, \\
\mathrm{II} & =\frac{1}{2^{n}} \sum_{k=1}^{\left[2^{n}\right]} T\left(\frac{k-1}{2^{n}}\right) E_{x}\left\{\frac{M\left(2^{-n}\right)-I}{2^{-n}}-B_{0}(x)\right\} \tilde{g}, \\
\mathrm{III} & =\frac{1}{2^{n}} \sum_{k=1}^{\left[2^{n}\right]} T\left(\frac{k-1}{2^{n}}\right)\left\{P T^{0}\left(2^{-n}\right) \tilde{g}-P \tilde{g}\right\}, \\
\mathrm{IV} & =\frac{1}{2^{n}} \sum_{k=1}^{\left[2^{n}\right]} T\left(\frac{k-1}{2^{n}}\right)\left\{E_{x}\left(\frac{M\left(2^{-n}\right)-I}{2^{-n}}\right)\left\{\tilde{g}\left(x\left(2^{-n}\right)\right)-\tilde{g}(x)-(B \cdot \nabla) \tilde{g}\right\}\right\},
\end{aligned}
$$


where $\tilde{g}=T^{0}(t-k \delta) \tilde{f}, \delta=2^{-n}$. The first term is clearly a Riemann sum for the right-hand side of (4.5). To estimate II, we note that

$$
\|\mathrm{II}\| \leqq \frac{1}{2^{n}} \sum_{k=1}^{\left[2^{n} t\right]} C\left(\frac{k-1}{2^{n}}\right) \varepsilon_{1}\left(\frac{1}{2^{n}}\right)\|\tilde{f}\| \leqq \varepsilon_{1}\left(2^{-n}\right)\left(\int_{0}^{t} C(s) d s\right)\|\tilde{f}\| .
$$

By assumption (4.1), $\lim _{\delta \rightarrow 0} \varepsilon_{1}(\delta)=0$.

To estimate III, we write $P T^{0}(\delta) \tilde{g}-P \tilde{g}=\frac{1}{2} P \int_{0}^{\delta} T^{0}(u) \Delta \tilde{g} d u$; thus

$$
\begin{aligned}
\|\mathrm{III}\|_{2} & \leqq \frac{1}{2^{n}} \sum_{k=1}^{\left[2^{\left.n_{t}\right]}\right.} C\left(\frac{k-1}{2^{n}}\right) \frac{1}{2}\left(\int_{0}^{2-n} K(u) d u\right)\|\Delta \tilde{g}\| \\
& \leqq \frac{1}{2}\left(\int_{0}^{2^{-n}} K(u) d u\right)\left(\int_{0}^{t} C(s) d s\right)\|\Delta \tilde{f}\| .
\end{aligned}
$$

Since $K \in L^{1}$, the first factor goes to zero when $n \rightarrow \infty$.

Finally, to estimate IV, note that if $x, y \in R^{n}, \tilde{g}(y)-\tilde{g}(x)=(y-x) \cdot \nabla \tilde{g}(x)$ $+\left\langle y-x, D^{2} \tilde{g}(\xi)(y-x)\right\rangle$, where $D^{2} \tilde{g}$ denotes the Hessian of $\tilde{g}$ and $\xi$ is a point on the line segment joining $x$ and $y$. Thus the last curly brace in IV equals $((x(\delta)-x)-B) \cdot \nabla \tilde{g}(x)+$ a term $\leqq|x(\delta) x|^{2}\left|D^{2} f\right|_{\infty}$. Hence

$$
\begin{aligned}
|\mathrm{IV}| & \leqq \frac{1}{2^{n}} \sum_{k=1}^{\left[2^{n} t\right]} C\left(\frac{k-1}{2^{n}}\right)\left\{E_{x}\left[\frac{M\left(2^{-n}\right)-I}{2^{-n}}\left(x\left(2^{-n}\right)-x\right)-B(x)\right] \cdot \nabla \tilde{g}\right. \\
& \left.\quad+\left|D^{2} f\right|_{\infty} E_{x}\left[\frac{M\left(2^{-n}\right)-I}{2^{-n}}\left|x\left(2^{-n}\right)-x\right|^{2}\right]\right\} \\
& \leqq \varepsilon_{2}\left(2^{-n}\right)\left(\int_{0}^{t} C(s) d s\right)|\nabla \tilde{f}|_{2}+(d(d+2))^{1 / 2} \varepsilon_{3}\left(2^{-n}\right)\left|D^{2} \tilde{f}\right|\left(\int_{0}^{t} C(s) d s\right)
\end{aligned}
$$

where we have used condition (4.1) in the first term. On the second term we have used the Schwarz inequality on the expectation, followed by condition (c) of the theorem and the evaluation $E_{x}|x(\delta)-x|^{4}=d(d+2) \delta^{2}$. The lemma is now proved.

(4.6) Lemma. The integral equation (4.5) has a unique solution $t \rightarrow T(t)$ which is a bounded operator on $\tilde{L}$.

Proof. If $T_{1}(t), T_{2}(t)$ are two solutions, we have for $\tilde{f} \in \tilde{L}$,

$$
\begin{gathered}
{\left[T_{1}(t)-T_{2}(t)\right] \tilde{f}=\int_{0}^{t}\left(T_{1}(s)-T_{2}(s)\right) P T^{0}(t-s) \tilde{f} d s,} \\
\left\|T_{1}(t)-T_{2}(t)\right\|_{\mathrm{op}}\|\tilde{f}\| \leqq \int_{0}^{t}\left\|T_{1}(s)-T_{2}(s)\right\|_{\mathrm{op}} K(t-s)\|\tilde{f}\| d s .
\end{gathered}
$$

Thus we have the inequality $F(t) \leqq \int_{0}^{t} F(s) K(t-s) d s$, where

$$
F(t)=\left\|T_{1}(t)-T_{2}(t)\right\|_{\text {op }}
$$

By simple iteration it follows that $F(t) \equiv 0$.

Proof of the Theorem. It now follows that if $t \rightarrow M(t)$ is a MOF of $(X, L)$ satisfying the hypotheses of the theorem, we have that, for $\tilde{f} \in \tilde{L}, E_{x}[M(t) \tilde{f}(x(t))]$ 
is the same as if $M$ were constructed according to the Itô equation of $\S 3$. But now by the multiplicative property, the same holds true for

$$
E_{x}\left[M(t) f(x(t)) g_{1}\left(x\left(t_{1}\right)\right) g_{2}\left(x\left(t_{2}\right)\right) \cdots g_{r}\left(x\left(t_{r}\right)\right)\right]
$$

where $0 \leqq t_{1}<t_{2}<\cdots<t_{r} \leqq t$, and $g_{1}, \ldots, g_{r}$ are scalar $L^{2}$ functions. By standard measure-theoretic arguments, such $g$ 's generate all of $\mathscr{F}_{t}$. Thus if $G \in \mathscr{F}_{t}, E_{x}[M(t) G]$ is the same as if $M$ were constructed according to the Itô equation of §3. But $M(t) \in \mathscr{F}_{t}$. Hence for each $x, M(t)$ is almost surely given by the Itô equation of $\S 3$. Finally, by the continuity of $M$, we have, for each $x$,

$$
P_{x}\left[M(t)=M_{1}(t), t \geqq 0\right]=1 .
$$

5. Martingale multiplicative functionals. The smoothness assumptions (4.1) can be partially dispensed with if we assume the normalization

$$
E_{x}[M(t)]=I
$$

where $I$ is the identity operator on $L$. Coupled with the multiplicative property, this implies that $M$ is a martingale relative to the fields $\left\{\mathscr{F}_{t}\right\}$. The natural tool to use here is the martingale stochastic integral, introduced by $\mathrm{H}$. Kunita and S. Watanabe [4], and further developed by P. A. Meyer [5] and his students. The main result is

(5.2) ThEOREM. Let $t \rightarrow M(t)$ be a continuous $\mathscr{M}$-valued MOF of $(X, L)$ where $X$ is a one-dimensional Wiener process and $L$ is a separable Hilbert space such that

(a) $\sup _{x \in R ; 0 \leqq t \leqq T} E_{x}\left[\|M(t)\|_{2}^{2}\right]=C(T)<\infty$,

(b) (5.1) holds,

(c) $M(t)$ is invertible for each $t \geqq 0$.

Then there exists an $\mathscr{M}$-valued function $x \rightarrow B(x)$, locally square integrable, such that $P_{x}\left[M(t)=M_{1}(t), t \geqq 0\right]=1$ where $M_{1}$ is the solution of

$$
M_{1}(t)=I+\int_{0}^{t} M_{1}(s) B(x(s)) d x(s),
$$

constructed in (3.7).

To prepare the apparatus for the proof, let us first find the increasing process associated with $M$. We use the following general fact.

(5.3) LEMMA. Let $\left\{M(t), \mathscr{F}_{t}, t \geqq 0\right\}$ be a continuous martingale with values in $a$ Hilbert space $\mathscr{M}$, such that $E\left[\|M(t)\|^{2}\right]<\infty$ for each $t \geqq 0$. Then there exists a real, increasing $\mathscr{F}_{t}$ adapted process $\{A(t), t \geqq 0\}$ such that

$$
E\left[\|M(t+s)-M(t)\|^{2} \mid \mathscr{F}_{t}\right]=E\left[A(t+s)-A(t) \mid \mathscr{F}_{t}\right], \quad 0 \leqq t<t+s .
$$

Proof. Let $\left\{\varphi_{k}\right\}_{k \geqq 1}$ be an orthonormal basis for $L ; M_{k}(t)=\left\langle M(t), \varphi_{k}\right\rangle$ is a real, locally square integrable martingale, and hence by a theorem of Meyer [6], there exists an increasing adapted process $A_{k}(t)$ such that

$$
E\left[\left\|M_{k}(t+s)-M_{k}(t)\right\|^{2} \mid \mathscr{F}_{t}\right]=E\left[A_{k}(t+s)-A_{k}(t) \mid \mathscr{F}_{t}\right]
$$


set $A(t)=\sum_{k \geqq 1} A_{k}(t) . A(t)$ is well defined since $\sum_{k \geqq 1} E A_{k}(t)=\sum_{k \geqq 1} E\left\|M_{k}(t)\right\|^{2}$ $=E\|M(t)\|^{2}<\infty$. Now if $B$ is any $\mathscr{F}_{t}$ measurable set, we have

$$
\begin{aligned}
E\left[(M(t+s)-M(t))^{2} I_{B}\right] & =\sum_{k \geqq 1} E\left[\left\langle M(t+s)-M(t), \varphi_{k}\right\rangle^{2} \cdot I_{B}\right] \\
& =\sum_{k \geqq 1} E\left[\left(A_{k}(t+s)-A_{k}(t)\right) \cdot I_{B}\right] \\
& =E\left[(A(t+s)-A(s)) I_{B}\right] .
\end{aligned}
$$

Hence (5.4) is proved.

We first define the stochastic integral $\int_{0}^{t} f(s) d M(s)$ where $M$ satisfies the conditions of Lemma (5.3) and $f$ is a real adapted process such that

$$
E \int_{0}^{t} f(s)^{2} d A(s)<\infty
$$

for each $t \geqq 0$. Indeed, if $f(t)=f\left(\left[2^{n} t\right] 2^{-n}\right)$, for some $n \geqq 1$, set

$$
\begin{aligned}
\int_{0}^{t} f(s) d M(s)= & \sum_{\left.0 \leqq k \leqq 2^{n} t\right]} f\left(k 2^{-n}\right)\left[M\left((k+1) 2^{-n}\right)-M\left(k 2^{-n}\right)\right] \\
& +f\left(\left[2^{n} t\right] 2^{-n}\right)\left[M(t)-M\left(\left[2^{n} t\right] 2^{-n}\right)\right] .
\end{aligned}
$$

It is easy to check that this definition does not depend upon $n$. For $\operatorname{such} f$, we can use the evaluation (5.4) to check that

$$
E\left\|\int_{0}^{t} f(s) d M(s)\right\|^{2}=E \int_{0}^{t} f(s)^{2} d A(s)
$$

Thus the mapping $f \rightarrow \int_{0}^{t} f(s) d M(s)$ is an isometry if we use the norm (5.5) on $f$. It is easy to show that any $f$ satisfying (5.5) can be approximated in this norm by $f$ 's for which the stochastic integral is already defined. Thus we can extend the mapping $f \rightarrow \int_{0}^{t} f(s) d M(s)$ by an abstract completion using (5.6).

In the applications below, the condition (5.5) is too stringent. We need to consider the class of adapted mappings $t \rightarrow f(t)$, such that

$$
P\left[\int_{0}^{t} f(s)^{2} d A(s)<\infty\right]=1 .
$$

Let $T_{n}=\inf \left\{t \geqq 0: \int_{0}^{t} f(s)^{2} d A(s) \geqq n\right\}$. According to the condition (5.7), there exists an integer $N=N(\omega)$ such that $T_{n}=+\infty$ for $n \geqq N(\omega)$. For any $n<\infty$ we have

$$
E\left\|\int_{0}^{t} f(s) I_{\left[0, T_{n}\right]}(s) d M(s)\right\|^{2}=E \int_{0}^{t} f(s)^{2} I_{\left[0, T_{n}\right]}(s) d s \leqq n .
$$

Thus the stochastic integral $\int_{0}^{t \wedge T_{n}} f(s) d M(s)=\int_{0}^{t} f(s) I_{\left[0, T_{n}\right]}(s) d M(s)$ is well defined for $n=1,2, \ldots$ and independent of $n$ for $n \geqq N(\omega)$.

Thus we can define $\int_{0}^{t} f(s) d M(s)$ as the trivial limit of $\int_{0}^{t \wedge T_{n}} f(s) d M(s)$, when $n \rightarrow \infty$. We can summarize the above as a

(5.8) Proposition. Let $\left\{M(t), \mathscr{F}_{t}, t \geqq 0\right\}$ be a continuous martingale with values 
in a Hilbert space $\mathscr{M}$ such that $E\left[\|M(t)\|^{2}\right]<\infty$ for each $t \geqq 0$. Let $t \rightarrow f(t)$ be an $\mathscr{F}_{t}$ adapted mapping such that $\int_{0}^{t} f(s)^{2} d A(s)<\infty$ almost surely, where $\{A(t), t \geqq 0\}$ is the increasing process constructed in Lemma (5.3). Then we can define the stochastic integral $\int_{0}^{t} f(s) d M(s)$ as a locally square integrable martingale satisfying the estimate (5.6), whenever the right-hand side there is finite.

We now return to the case at hand, where $\mathscr{M}$ is the Hilbert space of all HilbertSchmidt operators over $L$, and $M$ arises as a MOF of $(X, L)$ satisfying (5.1), where $X$ is a one-dimensional Wiener process. If $t \rightarrow H(t)$ is an $\mathscr{M}$-valued mapping, adapted to $\left\{\mathscr{F}_{t}\right\}$ and satisfying

$$
P_{x}\left[\int_{0}^{t}\|H(s)\|^{2} d A(s)<\infty\right]=1
$$

we can define the stochastic integral $\int_{0}^{t} H(s) d M(s)$ by the formula

$$
\int_{0}^{t} H(s) d M(s)=\sum_{k, l \geqq 1} \varphi_{k} \otimes \varphi_{l} \int_{0}^{t}\left\langle H(s) \varphi_{k}, \varphi_{l}\right\rangle d M(s)
$$

where $\left\{\varphi_{k}\right\}$ is a complete orthonormal set in $L$. It is easily checked that this expression is independent of the choice of basis.

Proof of Theorem (5.2). Let $H(t)=M(t)^{-1}$ in (5.9) and let us form the stochastic integral $C(t)=\int_{0}^{t} M(s)^{-1} d M(s)$, an element of $\mathscr{M}$. For $0 \leqq t<t+s$ we have

$$
\begin{aligned}
C(t+s)-C(t) & =\int_{0}^{s} M(t+u)^{-1} d_{u} M(t+u)=\int_{0}^{s} M(t+u)^{-1} M(t) d_{u} M\left(u, \theta_{t}\right) \\
& =\int_{0}^{s} M\left(u, \theta_{t}\right)^{-1} d_{u} M\left(u, \theta_{t}\right) \\
& =\left(\int_{0}^{s} M(u)^{-1} d M(u)\right) \cdot \theta_{t} \\
& =C\left(s, \theta_{t}\right) .
\end{aligned}
$$

Thus $\{C(t), t \geqq 0\}$ is a continuous additive functional of the Wiener path; furthermore $C\left(t \wedge \tau_{n}\right)$ has mean zero, where $\tau_{n}=\inf \{t:|x(t)|=n\}$. Hence, by a theorem of H. Tanaka [10], $C(t)$ admits the representation

$$
C(t)=\int_{0}^{t} B(x(s)) d x(s)
$$

for some $\mathscr{M}$-valued function $x \rightarrow B(x)$, such that $\int_{K}\|B(x)\|_{2}^{2} d x<\infty$ for each compact set $K$.

Now we claim that

$$
\int_{0}^{t} M(s) d C(s)=M(t)-I
$$

Indeed, if $f$ is a simple, real, adapted process, we have 


$$
\begin{aligned}
\int_{0}^{t} f(s) d C(s)= & \sum_{k=0}^{\left[2^{n} t\right]} f\left((k-1) 2^{-n}\right) \int_{(k-1) 2^{-n}}^{k 2^{-n}} M^{-1}(s) d M(s) \\
& +f\left(\left[2^{n} t\right] 2^{-n}\right) \int_{\left[2^{n} t\right] 2^{-n}}^{t} M^{-1}(s) d M(s) \\
= & \sum_{k=0}^{\left[2^{n} t\right]} \int_{(k-1) 2^{-n}}^{k 2^{-n}} f\left((k-1) 2^{-n}\right) M^{-1}(s) d M(s) \\
& +f\left(\left[2^{n} t\right] 2^{-n}\right) \int_{\left[2^{n} t 2^{-n}\right.}^{t} M^{-1}(s) d M(s) \\
= & \sum_{k=0}^{\left[2^{n} t\right]} \int_{(k-1) 2^{-n}}^{k 2^{-n}} f(s) M^{-1}(s) d M(s) \\
& +f\left(\left[2^{n} t\right] 2^{-n}\right) \int_{\left[2^{n} t\right] 2^{-n}}^{t} M^{-1}(s) d M(s) \\
= & \int_{0}^{t} f(s) M^{-1}(s) d M(s)
\end{aligned}
$$

by continuity, the equality between the two extreme members holds also for all $f$ 's satisfying (5.5), as well as those satisfying the more general (5.7). The definition (5.9) further extends this to $\mathscr{M}$-valued functions $s \rightarrow H(s)$. Now let $H(s)=M(s)$. This yields (5.11) and hence we have

$$
M(t)=I+\int_{0}^{t} M(s) d C(s)=I+\int_{0}^{t} M(s) B(x(s)) d x(s)
$$

which was to be proved. This completes the proof of Theorem (5.2).

REMARK. The above proof depends only on being able to give a reasonable definition of the stochastic integral $\int_{0}^{t} M^{-1}(s) d M(s)$. Thus, we only need to assume that $\{M(t), t \geqq 0\}$ is a semimartingale, i.e. $M(t)=M_{0}(t)+\tilde{M}(t)$, where $M_{0}$ is a martingale and $t \rightarrow \tilde{M}(t)$ is of bounded variation. By reasoning analogous to C. Doléans-Dade, [2], we can show that $C(t)=\int_{0}^{t} M^{-1} d M$ is again a semimartingale as well as an additive functional of $X$, and hence obtain (1.3) with $B_{0} \neq 0$.

\section{REFERENCES}

1. D. G. Babbitt, Wiener integral representations for certain semigroups which have infinitesimal generators with matrix coefficients, J. Math. Mech. 19 (1970), 1051-1067.

2. C. Doléans-Dade, Quelques applications de la formule de changement de variables pour les semimartingales, Z. Wahrscheinlichkeitstheorie und Verw. Gebiete 16 (1970), 181-194.

3. K. Itô and S. Watanabe, Transformation of Markov processes by multiplicative functionals, Ann. Inst. Fourier (Grenoble) 15 (1965), fasc. 1, 13-30. MR 32 \#1755.

4. H. Kunita and S. Watanabe, On square integrable martingales, Nagoya Math. J. 30 (1967), 209-245. MR 36 \#945.

5. P. A. Meyer, Séminaire de probabilités. Vol. I (Université de Strasbourg, 1966-67), Lecture Notes in Math., vol. 39, Springer-Verlag, Berlin, 1967.

6. - Probability and potentials, Blaisdell, Waltham, Mass., 1966; French ed., Publ. Inst. Math. Univ. Strasbourg, no. 14, Actualités Sci. Indust., no. 1318, Hermann, Paris, 1966. MR 34 \#5118; MR 34 \#5119. 
7. H. P. McKean, Jr., Stochastic integrals, Probability and Math. Statist., no. 5, Academic Press, New York, 1969. MR 40 \#947.

8. M. Pinsky, Multiplicative operator functionals of a Markov process, Bull. Amer. Math. Soc. 77 (1971), 377-380.

9. D. W. Stroock, On certain systems of parabolic equations, Comm. Pure Appl. Math. 23 (1970), 447-457.

10. H. Tanaka, Note on continuous additive functionals of the 1-dimensional Brownian path, Z. Wahrscheinlichkeitstheorie und Verw. Gebiete 1 (1962/63), 251-257. MR 29 \#6559.

11. A. D. Wentsell (A. D. Ventcel'), On continuous additive functionals of a multidimensional Wiener process, Dokl. Akad. Nauk SSSR 142 (1962), 1223-1226=Soviet Math. Dokl. 3 (1962), 264-266. MR 27 \#4265.

Department of Mathematics, Northwestern University, Evanston, Illinois 60201 